\title{
Getting ready for the micro-arcsecond era
}

\author{
A. G. A. Brown \\ Sterrewacht Leiden, Leiden University, \\ P.O. Box 9513, 2300 RA Leiden, The Netherlands \\ email: brown@strw.leidenuniv.nl
}

\begin{abstract}
As the title of this symposium implies, one of the aims is to examine the future of astrometry as we move from an era in which thanks to the Hipparcos Catalogue everyone has become familiar with milliarcsecond astrometry to an era in which microarcsecond astrometry will become the norm. I will take this look into the future by first providing an overview of present astrometric programmes and how they fit together and then I will attempt to identify the most promising future directions. In addition I discuss the important conditions for the maximization of the scientific return of future large and highly accurate astrometric catalogues; catalogue access and analysis tools, the availability of sufficient auxiliary data and theoretical knowledge, and the education of the future generation of astrometrists.
\end{abstract}

Keywords. astrometry, catalogs, surveys

\section{Overview of astrometric programmes}

Figure 1 presents a graphical overview of past, present, and future astrometric programmes and the relationships between them. I have grouped a number of the programmes together. For example, 'wide-field CCD surveys' stands for astrometry obtained from observing programmes such as the Sloan Digital Sky Survey (SDSS) or the future Large Synoptic Survey Telescope (LSST, see Ivezić 2008). Similarly 'radio interferometry' stands for a number of astrometric programmes conducted with different VLBI networks. The overview in figure 1 is not intended to be complete but serves to illustrate the variety of astrometric programmes and how they fit together.

All astrometric programmes must ultimately be linked to the International Celestial Reference System (ICRS, the idealized barycentric coordinate system to which celestial position are referred) through procedures which involve a number of steps that depend on the details of the astrometric observing programme. For ground-based programmes where the observations are typically done over a small field of view the obtained relative parallaxes and proper motions are converted to an absolute scale through the observations of astrometric standards. In the case of missions like Hipparcos although the measured parallaxes and proper motions are absolute by design, the reference frame still has six degrees of freedom with respect to the ICRS (three orientation and three spin parameters) which have to be taken out through observations of extra-galactic reference sources. The ICRS thus ties all astrometric programmes together and this is symbolized by the background image in figure 1 .

The practical realization of the ICRS is the International Celestial Reference Frame (ICRF) consisting of a few hundred extra-galactic radio sources with adopted ICRS positions. The ICRF sources are constantly monitored in dedicated VLBI observing programmes, which is why the radio interferometry observations are portrayed separately in figure 1 as they underpin the practical realization of the reference system. In the optical, the ICRF is materialized by the Hipparcos Catalogue. For more details see the chapters on reference frames in this volume. For Solar system investigations radar astrometric 


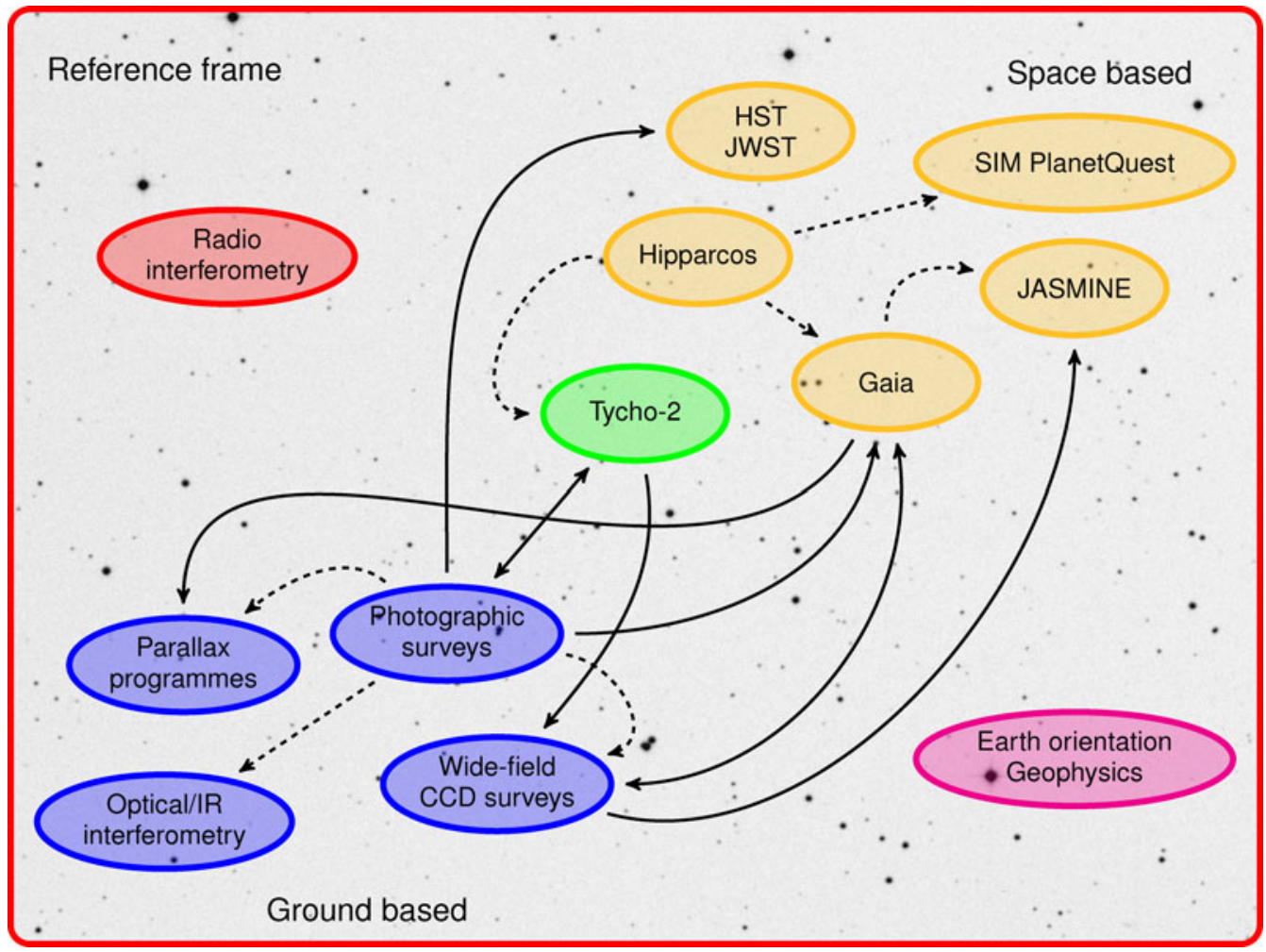

Figure 1. Graphical overview of astrometric programmes and the relations between them. There is no ordering in terms of timing or accuracy of the programmes. Refer to the text for further explanations of this figure. The background image, symbolizing the reference frame, is a $20 \times 15$ $\operatorname{arcmin}^{2}$ region of the sky centred on the Hyades cluster and was taken from the Digitized Sky Survey (POSS-II plates).

observations (ranging and Doppler) are instrumental in providing the reference for accurate ephemerides of the inner planets and the determination of various astronomical constants (see Pitjeva in this volume).

A well established reference system can be used to interpret accurate observations of celestial sources from earth in terms of the orientation of the earth's spin axis and its precession and nutation (bottom right of figure 1). Two particularly interesting examples are given in the contributions by Vondrák and Huang in this volume. The former concerns a study of earth rotation over the past 100 years and the latter a study of the precession and nutation of the earth's rotation axis in terms of a non-rigid model of our planet.

The other astrometric programmes in figure 1 have been lumped together into the categories 'ground based' and 'space based', with the Tycho-2 catalogue located in between as that was constructed from a combination of photographic plate material and observations made with the Hipparcos satellite. The arrows illustrate some of the relations within the ground based or space based programmes (dashed lines) and between ground based and space based astrometry (solid lines).

The photographic surveys that were carried out during the 20th century have formed the basis for the optical reference frame until the Hipparcos catalogue was published and the astrometric catalogues derived from them, now reduced to the Hipparcos reference frame, still serve as basic input for modern ground based astrometric programmes. Recently the photographic material has been combined with CCD astrometric observa- 
tions such as in the UCAC catalogues (Zacharias et al. 2004). Eventually the wide field CCD surveys will provide a much denser optical reference frame, although they will still have to be linked to more absolute frames, e.g. Hipparcos or Gaia.

A good example of how ground based astrometric surveys support the space missions is the Guide Star Catalogue (GSC, Bucciarelli 2008). It serves as the basic input for HST pointing and guidance and will also form the basis of the input catalogue for JWST. For Gaia the GSC will be one of the inputs for the construction of an initial source list to support the identification of sources in the early phases of the mission. The latest version of the GSC makes use of Tycho-2 data as a reference for the astrometric calibration and as a supplement to its bright end.

The ground based CCD surveys go much deeper than missions like Gaia or JASMINE will and the corresponding astrometric catalogues can serve to provide information on sources beyond the survey limits of these missions. This is important in regions on the sky where the density is high enough for faint sources to disturb the astrometry and photometry of the target sources. Conversely, the ground based CCD surveys can make use of the reference frame provided by space based missions. The Tycho- 2 catalogue is already being used for this purpose and in the future the Gaia and SIM PlanetQuest catalogues will provide an excellent reference, in particular for the ground based parallax programmes (Smart 2008).

Finally, the space astrometry missions are also interrelated. The Hipparcos catalogue has been used to select candidate grid stars for SIM PlanetQuest (see e.g. Hekker et al. 2006) and will be used to predict the positions of sources on the sky that are too bright to be observed by Gaia. The latter will affect the measurements of nearby fainter sources and will influence the state of Gaia's CCDs with respect to charge transfer inefficiency (see Lindegren 2008). The JASMINE mission will rely on a plate overlap technique and observe only a relatively small part of the sky. Thus this mission will benefit greatly from the reference frame which will be provided by Gaia.

In summary all the astrometric programmes schematically indicated in figure 1 form a very tightly interlocked set of observational programmes, none of which be obsolete for some time to come. In the next section I will look at how these astrometric programmes cover the space of survey parameters and what science is consequently not well covered.

\section{Science covered by astrometric surveys: are we missing something?}

Astrometric programmes can be characterized by the following parameters:

Precision and accuracy: These are the most obvious selling points of any astrometric survey and much effort goes into maximizing these parameters. The precision reflects the quality of the relative measurements that can be made with a particular instrument and optimizing this parameter is enough if one is only interested in differential measurements, such as when hunting for exoplanets. The accuracy of a survey is limited by the external calibration of the measurements and by the design of the experiment. A well known example is the case of ground-based parallax programmes which are done over small fields of view and thus require corrections of precisely measured relative parallaxes to absolute ones. The accuracy in this case is limited by our knowledge of the spatial distribution of the reference sources.

Time baseline: Parallax and proper motion measurements benefit from a long time baseline, which is needed to disentangle the parallaxes from the proper motions and to ensure that proper motions of binary systems reflect the centre of mass motion as opposed to the orbital motions of the components. The sampling in time also plays an important role, especially for parallax measurements carried out over only a few years. 


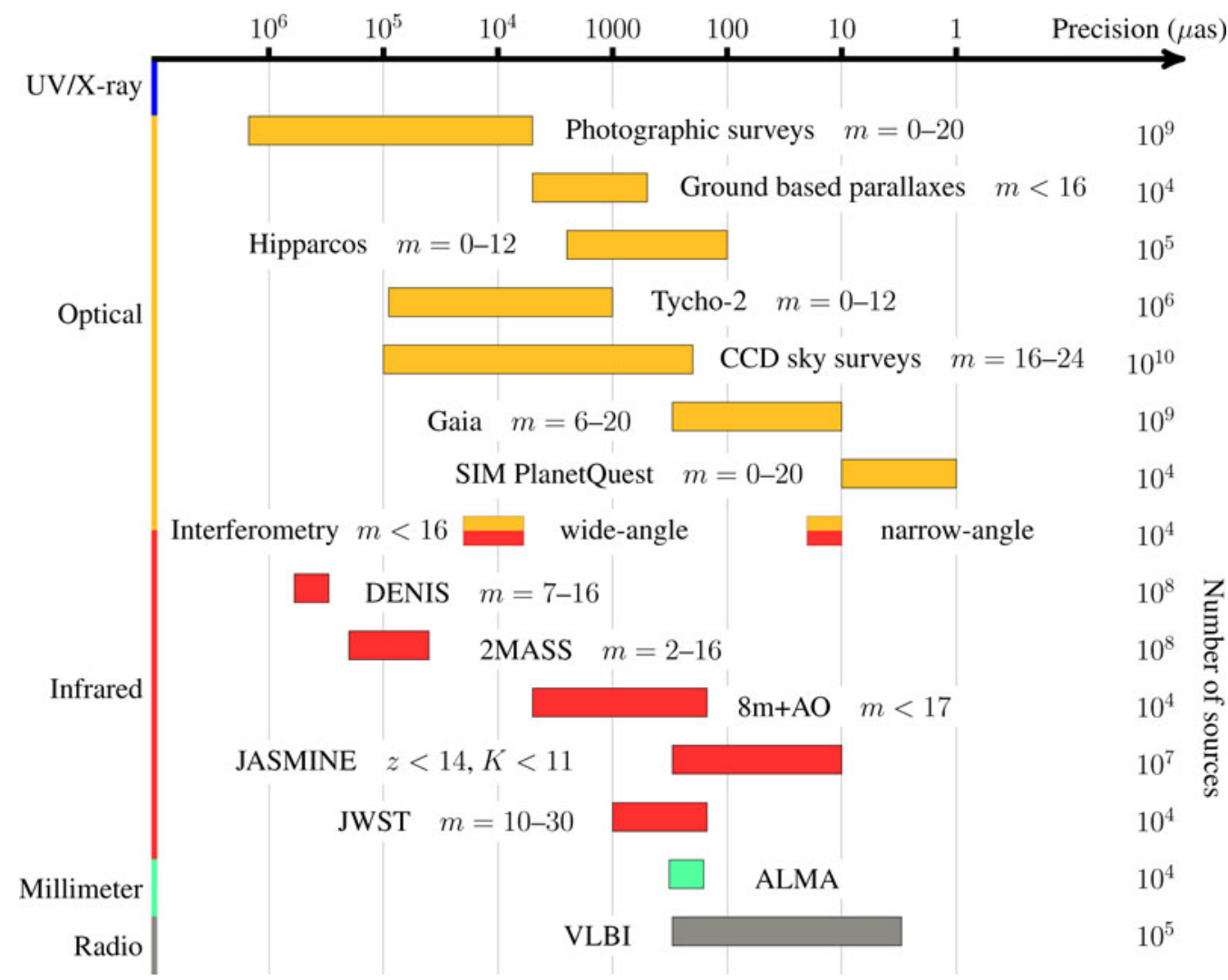

Figure 2. Graphical overview of astrometric surveys. The shading of the boxes and the right vertical axis serves to aid the eye in segregating the wavelength domains. Refer to the text for further explanation of the diagram. The survey parameters are based on the following references: Photographic surveys - Morrison et al. (2001), Monet et al. (2003); Ground based parallaxes - Jao (2008), Smart (2008); Hipparcos — ESA (1997), van Leeuwen (2007); Tycho-2 - Høg et al. (2000); CCD sky surveys - Ivezić (2008), Magnier (2008); Gaia - Lindegren (2008); SIM PlanetQuest - Shao (2008); Optical/IR interferometry — Boden (2008), Richichi (2008); DENIS - Borsenberger et al. (2006), G. Simon priv. comm.; 2MASS - Skrutskie et al. (2006); 8m+AO Röll et al. (2008), Ghez (2008); JASMINE — Gouda (2008); JWST — Beichman (2008); ALMA — Lestrade (2008); VLBI - Reid (2008), Ma (2008).

Depth and dynamic range: The faint limit of the survey determines the kinds of sources that can be observed (faint nearby low-mass objects, distant standard candles, extra-galactic reference sources, etc). Future astrometric surveys will push this parameter as much as possible but it has to be traded off against the dynamic range of the survey. Instruments capable of observing very faint sources often cannot deal with very bright sources. For example the Hipparcos astrometry for the brightest $(V<6)$ stars will not be superseded by any of the planned space missions. On the other hand optical/infrared interferometry programmes can typically only deal with bright sources.

Number of sources: For sources of arbitrary brightness achieving high precision always comes at the expense of more observing time and so a trade-off has to be made between the number of sources observed and the astrometric precision achieved. Targeted high precision programmes (such as SIM or the ground-based parallaxes) are very good at addressing specific scientific questions whereas less precise 'all-sky' sur- 
veys are scientifically important because of the advantages of statistical studies of large samples and they come with the added bonus of serendipity.

Density of sources: The density of sources is important if the astrometric catalogue is to be used as a reference frame for other astrometric programmes. This is especially needed for astrometric surveys targeting faint sources over small fields of view.

Wavelength range: This parameter sets the 'window on the universe' for an astrometric survey and is a very important driver for the science that one can do.

Narrow- vs. wide-angle: In ground-based astrometry programmes sub-milliarcsec to micro-arcsec precision can be achieved but only through differential measurements over small fields. This makes it difficult to construct reference frames free from zonal systematic errors and necessitates the correction from relative to absolute parallaxes. On the contrary astrometry over wide angles by design gives absolute parallaxes and a rigid reference frame (e.g. Lindegren 2005). The problem of converting relative to absolute astrometry can in principle be overcome for narrow angle measurements if the observations go deep enough to find enough suitable extra-galactic reference sources in the field of view.

Ground based vs. space based: This parameter is closely related to the previous one. The choice of going to space offers the advantages of a stable thermal environment, freedom from gravity and the atmosphere, and full-sky visibility (Lindegren 2005). These factors in fact enable wide-angle astrometry as implemented on missions such as Hipparcos, Gaia, and SIM PlanetQuest.

Figure 2 is my attempt at summarizing the coverage of the parameter space described above by the present and planned astrometric catalogues and surveys. The main goal of this diagram is to identify major gaps in the parameter space and not to provide a complete and precise overview of all astrometric surveys or programmes. Figure 2 shows the distribution of astrometric programmes over wavelength, precision, depth and dynamic range, and the number of sources observed. The precision figures encompass positions, parallaxes and proper motions and in most cases also reflect the accuracy of the survey. Precision numbers for proper motions are generally numerically smaller than those for positions, especially for photographic surveys. The magnitude limits and numbers of objects are crude indicators. Within each wavelength range the surveys have been ordered roughly in time. From figure 2 a number of observations can be extracted which can help in identifying interesting future directions for astrometry.

In the optical we can expect an enormous amount of astrometric data, with accuracies in the 10 microarcsec to 10 milliarcsec range, coming in over the next 10 to 15 years from the CCD surveys of the sky (LSST, Pan-Starrs) and Gaia. A preview of what we can expect from this data is given in the contribution of Ivezic in this volume in which he discusses the power of using SDSS Data Release 6 for studies of the structure of the Milky Way. The latest SDSS data release also contains proper motions with $\sim 3-6$ mas $/$ yr errors out to $r=20$ for $30 \times 10^{6}$ stars (Munn et al. 2004). This offers photometry for a huge number of stars over a large volume in our galaxy combined with kinematic data at a level of accuracy which enables both a quantitative and qualitative breakthrough. This dataset will be dwarfed by the combined Gaia/LSST/Pan-Starrs data which will constitute Hipparcos quality astrometry over the magnitude range 20-24!

The ground-based parallax programmes and the optical/IR interferometric observations will fill very important niches at the bright end in the characterization of the Sun's nearest neighbours and in the hunt for exoplanets.

From figure 2 it is very clear that the SIM PlanetQuest mission is unique in its capability of providing astrometry in the 1-10 $\mu$ as range all the way out to 20 th magnitude. It 
will thus be the only instrument capable of directly addressing detailed questions about the outer reaches of our galaxy or nearby external galaxies.

All the optical programmes obviously suffer from the extinction barrier due to dust, especially in the Milky Way disk and towards the Galactic centre. Infrared surveys are thus essential in achieving a complete understanding of our galaxy and for studying star formation in all its aspects. In the infrared the astrometric data is currently of relatively poor quality compared to what is available in the optical even though the DENIS and 2MASS surveys represent a tremendous step forward compared to the situation 10 years ago. The only ongoing astrometric programmes in the infrared are the interferometric and adaptive optics programmes which mainly deliver relative astrometry over small fields of view (although at high precision). The only planned future infrared astrometric survey is the JASMINE mission which will cover the Bulge and inner Milky Way disk regions.

A very exciting prospect in the near future is the possibility of obtaining milliarcsecond astrometry in the millimetre wavelength regime. Combined with the already available radio astrometry at this accuracy this will enable the direct determination of the distances and kinematics of deeply embedded star clusters or even proto-stellar clusters (see contributions by Loinard and Lestrade).

\section{Future directions}

From the discussion of parameter space coverage by current and planned astrometric programmes a number of gaps can be identified from which proposals for future astrometric observing programmes can be extracted. I briefly discuss here a number of possibilities, some of which are already covered by planned instruments.

All-sky infrared astrometry at high accuracy: JASMINE covers the inner Milky Way disk only and does not go very deep. I think a deep all sky astrometric survey in the infrared provides the biggest 'discovery space', offering a tremendous improvement in our knowledge of the Milky Way disk, spiral arms and star forming regions. In addition low mass stars, brown dwarfs and 'free-floating planets' can be studied over a much larger volume. Such a mission is probably most efficiently carried out as a scanning spacecraft using the principles of Hipparcos and Gaia. The question is whether it will be technically feasible for a reasonable cost. Can a large cryogenic instrument be realised which can operate over the length of time required for high precision astrometry? The experience from the design of missions like ESA's DARWIN will be very useful in this respect.

High-precision narrow angle astrometry: This is an area which is already under development through the interferometric and adaptive optics programmes at the current generation of large ( $8 \mathrm{~m}$ class) telescopes. Pushing the precision of small fields of view as much as possible towards the $\mu$ as mark will increase the sensitivity of exoplanet searches to the level where earth-sized planets around nearby stars can be discovered; allow us to probe space-time near the massive black hole in the centre of the Milky Way; and enable the study of stellar orbits around the central black holes in external galaxies. This increase in astrometric accuracy can be achieved with new instruments on $8 \mathrm{~m}$ class telescopes used as interferometers and with instruments for the future extremely large telescopes (see the contributions by Ghez and Richichi in this volume).

Geometric distances and motions of galaxies in the local volume: The study of the dynamics of galaxies in the local volume is currently limited by the lack of knowledge of their proper motions. As discussed in the contribution by Bruntahler proper motions can be obtained as far out as the M31 subgroup through VLBI measurements of masers with respect to background quasars. He also discusses the possibility to go 
beyond the local group. An important goal for the future is to extend these studies by improving the precision and the enlarging the sample.

Micro-arcsecond astrometry to 25th magnitude: An obvious future instrument to consider would be a 'super-Gaia', capable of delivering microarcsecond astrometry to 25th magnitude. This would require careful consideration of a number of issues. What will be gained scientifically? Is Hipparcos quality data to 24th magnitude enough? The answer to this question will depend on what we learn from Gaia and the CCD surveys. The technical difficulties of simply scaling up Gaia will be driven by the need to collect 2 to 4 orders of magnitude more photons over the magnitude range $20-25$. This cannot be achieved through more sensitive detectors (the Gaia CCDs already having $~ 80 \%$ quantum efficiency) but will have to come from larger optics and a larger field of view. This will make the requirements on instrument stability very difficult to achieve (thermal and mechanical design) if not impossible. It is probably better to think about alternative ways of efficiently doing all-sky absolute astrometry at the microarcsecond level.

Astrometry at wavelengths below $300 \mathrm{~nm}$ : I included the UV/X-ray wavelength regime in figure 2 to highlight that currently there is no high precision astrometry available shortward of optical wavelengths. It is not clear that efforts to acquire high accuracy astrometry in UV/X-ray domain will pay off. It is technically difficult and most UV/X-ray sources may have optical counterparts which can be measured astrometrically.

\section{Maximizing the scientific return}

Although thinking about future directions for astrometric surveys is interesting it is much more important to ensure that we optimize the scientific exploitation of the large amounts of high accuracy astrometric data we can expect over the next two decades. This concerns the following issues:

Complementary data: All the large surveys (Gaia, LSST, Pan-Starrs) will deliver multi-colour photometry which is essential in the scientific exploitation of the astrometric data. Photometry provides the astrophysical characterization of the stars observed and allows the derivation of photometric distances. The latter can be calibrated using Gaia data for bright stars and will provide more reliable distances for the faint stars than can be derived from parallaxes. In the case of Gaia, at the faint end of the survey the low resolution prism spectra will not have much discriminating power with respect to surface gravity and metallicity of the stars. This can be remedied (for most of the sky) by supplementing Gaia photometry with the data from LSST and Pan-Starrs. Hence collaboration between these projects is essential.

Phase space for stars and galaxies consists of six dimensions but in the surveys targeting the stars in our galaxy the 6th dimension, radial velocity, will not be measured for the majority of the $10^{10}$ stars for which astrometric data will be collected. The RAVE project (Steinmetz et al. 2006) will collect radial velocities for $\sim 10^{6}$ stars and Gaia will do so for $\sim 10^{8}$ stars. The rewards for gathering radial velocities for the remaining stars are substantial and we should think about efficient ways of doing so. The accuracies of the radial velocities should be matched to the astrometric accuracies (translated to linear velocities) which for the faint end of the surveys lead to rather modest requirements and which can be met by low resolution spectrographs optimized for faint stars. From the ground a radial velocity survey, even with large multi-object instruments, may be very difficult to complete for the whole sky to 20-24th magnitude and dedicated followup programmes to pursue specific questions appear more viable. From space an all-sky survey may be possible using a scanning satellite optimized for radial velocities.

An important issue concerning radial velocities is the accuracy of the zero-point of 
the velocity scale. Given the huge efforts to acquire highly accurate absolute astrometry, the radial velocities should be absolute to the same level of accuracy. This point has not been addressed over the last decade as most of the effort has gone into refining the relative precision of radial velocity surveys in order to optimize them for planet detection. A dedicated effort is needed now to improve the zero-point accuracy across the Hertzsprung-Russell diagram. Astrometric data from Gaia can be used for this through the astrometric radial velocity technique (Dravins, Lindegren, \& Madsen 1999) which provides radial velocities free of effects intrinsic to the star.

The compromise between resolution and depth of the spectroscopic surveys carried out by the RAVE project and Gaia is driven by the need for high resolution spectroscopy for the detailed determination of the astrophysical parameters of stars, including rotational velocities. Gathering complementary high resolution spectra is a very expensive undertaking and is probably best done with well designed follow-up projects that serve, for example, to better calibrate photometric indicators or answer specific questions.

The last remark on complementary data concerns the determination of accurate reddening laws and extinction towards the stars and galaxies observed. Especially for the subset of stars with very high accuracy parallaxes the knowledge of reddening and extinction will become a limiting factor in the determination of luminosities and stellar parameters and hence in the improvement of our understanding of stellar structure and evolution. There is a dedicated effort within the Gaia data processing consortium to ensure a proper determination of reddening and extinction. This effort will benefit greatly from collaboration with other survey projects and from investigating the use of complementary data such as infrared photometry and direct measurements of the gas and dust components of the Milky Way.

Preparing for the analysis of large astrometric/photometric catalogues: Optimizing the science return depends critically on having analysis tools and theoretical models that enable a proper interpretation of the vast quantities of high accuracy astrometric and photometric data. For stars the determination of the fundamental parameters luminosity, age, mass, and chemical composition depends heavily on the quality of stellar structure and atmosphere models. As discussed in the contributions by Lebreton and Chaboyer many uncertainties still remain in stellar models. Although addressing these problems will depend on the availability of highly accurate astrometric data, steps can already be taken now to improve our understanding of stars through better theoretical models, the development of 3D model atmospheres and laboratory experiments aimed at obtaining better opacities, thermonuclear reaction rates and equations of state.

For the interpretation of the astrometric data in terms of Galactic structure and dynamics analysis tools should be developed that can deal with data for $10^{9}$ stars. Specific consideration should be given to the way parallax data is treated in order to avoid the biases that can be introduced when converting relatively imprecise parallaxes to distances. The best way forward may be to design analysis methods that rely on predicting the observations of Galactic phase space rather than the phase space variables themselves.

Providing optimized access to large catalogues: If the data for $10^{9}-10^{10}$ stars were to land on one's desk today it would be very difficult to decide how to begin exploring these data and extracting interesting science. The accessibility of these large catalogues will be essential in determining their success. The catalogue producers should aim at making tools available that allow easy access trough both high-level, easy to use, and low-level, more complex, interfaces. This includes good visualization tools. The catalogues should not be static finished products but should allow users to re-process data based on newly acquired knowledge about certain objects or classes of objects (see for example the discussion by Pourbaix in this volume). Improved knowledge should be 

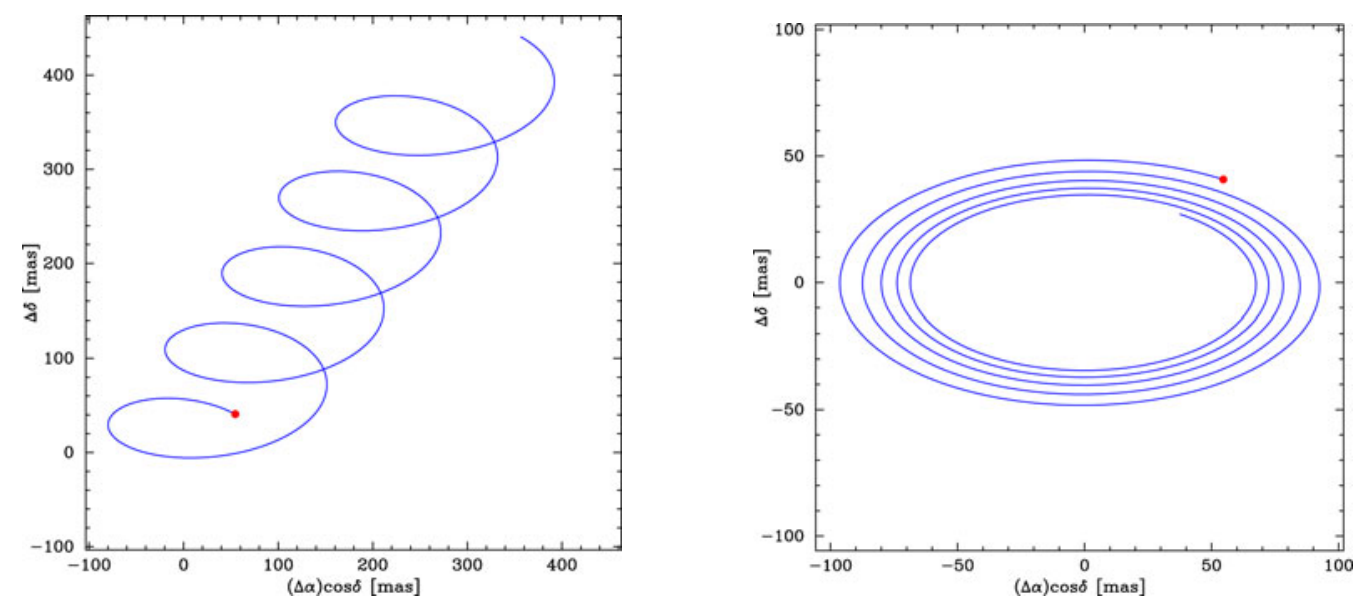

Figure 3. The paths on the sky for a star with a given proper motion and parallax (left) and for a star moving radially away from the Sun (right). This illustrates that from astrometry alone the $3 \mathrm{D}$ positions and motions of stars (including radial velocities) can be inferred. The right hand figure is not relevant in practice but as discussed in the paper by Dravins et al. (1999) there are a number of other astrometric effects from which radial velocities can be inferred. A practical application using Hipparcos data is given in Madsen et al. (2002). This figure was produced with the aid of the IAU's SOFA library (http://www.iau-sofa.rl.ac.uk/).

added to these databases when it becomes available. This could be, for example, radial velocities from follow-up programmes or a better astrophysical parametrization of stars based on improvements in stellar atmosphere models.

Users of the catalogues should be educated through documentation which has to be designed to be easy to understand for beginners while at the same time allowing experts to quickly navigate to the relevant details.

Training the next generation of astrometrists: The design and operation of astrometric programmes is a highly specialized undertaking and it is essential to ensure continuity of the available expertise in the astronomical community. This expertise should cover all aspects of carrying out astrometric programmes, whether they are scanning or interferometric space missions or ground based astrometry programmes, ranging from the establishment of the reference frame with VLBI observations to 'classical' parallax programmes. In addition astrometry at the microarcsecond level requires orders of magnitude better control over instrument calibration and the routine inclusion of effects such as general relativity in the data analysis. Without dedicated astrometry experts the whole edifice illustrated in figure 1 will fall apart. The experts are also needed to ensure a thorough understanding of the contents and the interpretation of astrometric catalogues. At this symposium a session was therefore dedicated to astrometry education, and I refer to the corresponding chapter in this volume for a summary.

\section{Final remarks}

I will conclude with a little 'pep-talk'. Astrometry is unfortunately considered to be a rather unglamourous discipline which is often even put aside as not being 'science'. I disagree with this; designing and carrying out high accuracy astrometric experiments is a highly scientific undertaking in itself and the results are of fundamental importance to astronomy, astrophysics and geodynamics. Astrometry tells us about our place in the universe, provides the only direct distance measurements to the stars, and can even be 
used to look inside our own planet as discussed by Huang in this volume. If we seek to motivate people to pursue a career in astrometry we should advertise that the beauty of this discipline, illustrated in figure 3 , lies in that fact that:

By carefully watching the positions of stars on the sky over the course of time you can find out (almost) all there is to know about the universe.

\section{Acknowledgements}

I thank Michael Perryman for his comments and Andreas Quirrenbach for reminding me that astrometric radial velocities are interesting in practice, not just in principle.

The background image in figure 1 was obtained from the Digitized Sky Surveys, which were produced at the Space Telescope Science Institute under U.S. Government grant NAG W-2166. The Second Palomar Observatory Sky Survey (POSS-II) was made by the California Institute of Technology with funds from the National Science Foundation, the National Geographic Society, the Sloan Foundation, the Samuel Oschin Foundation, and the Eastman Kodak Corporation. The Oschin Schmidt Telescope is operated by the California Institute of Technology and Palomar Observatory.

\section{References}

Beichman, Ch. 2008, this volume, p.238

Boden, A. F. 2008, this volume, p.36

Borsenberger, J., et al. 2006, in Visions for Infrared Astronomy, eds. V. Coudé du Foresto, D. Rouan, \& G. Rousset, Paris, RS série I2M vol. 6, Lavoisier

Bucciarelli, B. 2008, this volume, p.316

Dravins, D., Lindegren, L., \& Madsen, S. 1999, A\& A, 348, 1040

ESA 1997, The Hipparcos and Tycho Catalogues, ESA SP-1200

Ghez, A. M. 2008, this volume, p.52

Gouda, N. 2008, this volume, p.248

Hekker, S., Reffert, S., Quirrenbach, A., Mitchell, D. S., Fischer, D. A., Marcy, G. W., Butler, R. P. 2006, $A \mathscr{E} A, 454,943$

$\mathrm{H} \varnothing \mathrm{g}$, E., Fabricius, C., Makarov, V. V., et al. 2000, A\&\&A, 355, L27

Ivezić, Ž. 2008, this volume, p.537

Jao, W. 2008, this volume, p.421

Lestrade, J.-F. 2008, this volume, p.170

Lindegren, L. 2005, in The Three-Dimensional Universe with Gaia, eds. C. Turon, K. S. O'Flaherty \& M. A. C. Perryman, ESA SP-576, p.29

Lindegren, L. 2008, this volume, p.217

Ma, C. 2008, this volume, p.337

Madsen, S., Dravins, D., \& Lindegren, L. 2002, A\& A, 381, 446

Magnier, E. A. 2008, this volume, p.552

Monet, D. G., Levine, S. E., Canzian, B., et al. 2003, AJ, 125, 984

Morrison, J. E., Röser, S., McLean, B., et al. 2001, AJ, 121, 1752

Reid, M. 2008, this volume, p.141

Richichi, A. 2008, this volume, p.44

Röll, T., Seifahrt, T., Neuhauser, R. 2008, this volume, p.48

Skrutskie, M. F., Cutri, R. M., Stiening M. D., et al. 2006, AJ, 131, 1163

Shao, M. 2008, this volume, p.231

Smart, R. 2008, this volume, p. 429

Steinmetz, M., Zwitter, T., Siebert, A., et al. 2006, AJ, 132, 1645

van Leeuwen, F. 2007, Hipparcos, the New Reduction of the Raw Data, Springer

Zacharias, N., Urban, S. E., Zacharias, M. I., et al. 2004, AJ, 127, 3043 